\title{
ULTRASTRUCTURE AND ANTIGENS OF PARTICLES FROM RABBIT SEMEN*
}

\author{
GHARLES B. METZ, GERTRUDE W. HINSCH $\dagger$ AND \\ JUDITH L. ANIKA \\ Institute of Molecular Evolution, University of Miami, \\ Coral Gables, Florida
}

(Received 3rd April 1968)

Semen of the rabbit, unlike that of many mammals, normally contains a high concentration of particles in addition to the spermatozoa. These particles have received very little attention by students of the male reproductive products. The particles can be separated from the spermatozoa only with considerable difficulty. Therefore, they are hazards as contaminants of 'washed' spermatozoan suspensions and should be taken into account in biochemical, immunological and even fertilization studies on ejaculated rabbit spermatozoa. In addition, the nature of these particles, their origin and possible role in reproduction should be of some intrinsic interest. Accordingly, the ultrastructure and some immunological properties of the particles were examined.

Semen samples were obtained by means of an artificial vagina from Dutch Belted rabbits. Vasectomized males provided spermatozoon-free semen samples which served as the source of seminal particles.

For electron microscopy, seminal solids were concentrated by light centrifugation ( $\mathrm{g} \max =12,000 \mathrm{~g}$ for $10 \mathrm{~min}$ ). The supernatant was removed and the resulting pellet fixed in $5 \%$ glutaraldehyde in $0.05 \mathrm{M}$-cacodylate $-0.15 \mathrm{~m}$ sucrose buffer for $90 \mathrm{~min}$. The pellets were post-fixed for $1 \mathrm{hr}$ in $1 \% \mathrm{OsO}_{4}$ in the cacodylate-sucrose mixture, dehydrated in a graded alcohol-propylene oxide series and embedded in Araldite. In addition, seminal coagula containing entrapped particles were fixed. Sections were cut with glass or diamond knives on a LKB ultrotome or Potrer-Blum MT-2. The sections were mounted on formvar-coated grids, stained with alcoholic uranyl acetate and lead citrate and viewed with the Philips EM 200.

Examination of sectioned pellets of seminal solids from normal males showed spermatozoa, an occasional leucocyte and several kinds of 'particles' (P1. 1, Fig. 1). These particles are readily classified into at least three categories according to the character of their internal organization, namely dense, vesicular and amorphous. These same types are also found in micrographs of seminal coagula (Pl. 1, Fig. 2) and of pellets of seminal solids from vasectomized males (Pl. 1, Figs. 3 and 4). Particles of all three classes may be

* Contribution No. 101 from the Institute of Molecular Evolution. Aided by research grants from the National Science Foundation (No. GB-3899) and the National Aeronautics and Space Administration (Na-G-689).

$\dagger$ National Science Foundation Science Faculty Fellow. 
bounded by a limiting membrane. However, amorphous particles were frequently seen with imperfect or possibly disintegrating membranes.

Since most or all of the particles are present in ejaculates obtained from vasectomized males, they evidently are not of testicular or spermatozoan origin. The male accessory glands appear to be the most likely source. Possibly some of the particles are of cellular, nuclear or mitochondrial origin. However, none of the particles in the suspension fluoresced green when stained with acridine orange.

For most immunological tests the particles were centrifuged from the seminal fluid and washed one or more times in Hanks's Balanced Salt Solution (Microbiological Associates Inc., Bethesda, Maryland). Antisera were prepared in separate guinea-pigs against whole fertile rabbit semen, 'seminal solids' (washed centrifugate from fertile semen, e.g. spermatozoa plus seminal particles) and seminal plasma (supernatant from centrifuged semen). The immunizing antigen preparations were emulsified in an equal volume of Freund's complete adjuvant (Difco) and administered by intramuscular injection. The guinea-pigs were bled by cardiac puncture for control (pre-injection) and immune serum.

Ouchterlony agar gel precipitin tests were performed in $0.85 \%$ agar (Agarose, Bausch \& Lomb) containing borate buffered saline $(\mathrm{pH} 8.5)$ and $0.5 \%$ sodium azide (final concentration) as a preservative. Antigen and antibody wells were $6.0 \mathrm{~mm}$ apart on a $2 \times 2$ in. glass plate and contained $0.1 \mathrm{ml}$ of sample.

Seminal particles were examined for antigens by agglutination and precipitin tests, and the antigenic components were compared with those of seminal plasma. Spermatozoa and seminal particles frequently auto-agglutinated spontaneously (Bedford, 1965, for references). Since such auto-agglutination can be prevented by nigrosine (Dott \& Walton, 1960), agglutination tests were confirmed in experiments using $10.0 \%$ nigrosine (final concentration).

Seminal particles agglutinate readily when mixed with any of the three types of antisera employed, namely anti-whole semen, anti-seminal solids ( $\mathrm{Pl}$. 2, Fig. 5a) and anti-seminal plasma, whereas particles fail to agglutinate in control guinea-pig serum (Pl. 2, Fig. 5b). In addition, the particles in normal fertile semen agglutinate strongly when semen is mixed with antiserum. Accordingly, at least a large proportion of the seminal particles must possess surface antigens. Some particles are generally seen to be free from agglutinates in the antiserum-treated preparations so it is possible that one or more kinds of particles lack antigens.

\section{EXPLANATION OF PLATE 1}

FIG. 1. Seminal solids in normal semen. Seminal particles of the amorphous (a) and vesicular (v) type are evident. The disintegrating spermatozoan material at (I) may represent a phagocytic cell. $\times 14,750$.

Fig. 2. Section through seminal coagulum from a vasectomized male. (a) amorphous particle; (c) coagulum showing fine gel structure; (d) dense seminal particle. $\times 8333$. Fig. 3. Seminal solids from vasectomized rabbit. (a) amorphous particle; (d) dense particle; (v) vesicular particle. $\times 25,830$.

Fig. 4. Seminal solids from vasectomized rabbit. (a) amorphous particle; (d) dense particle. $\times 35,000$. 
PLATE 1

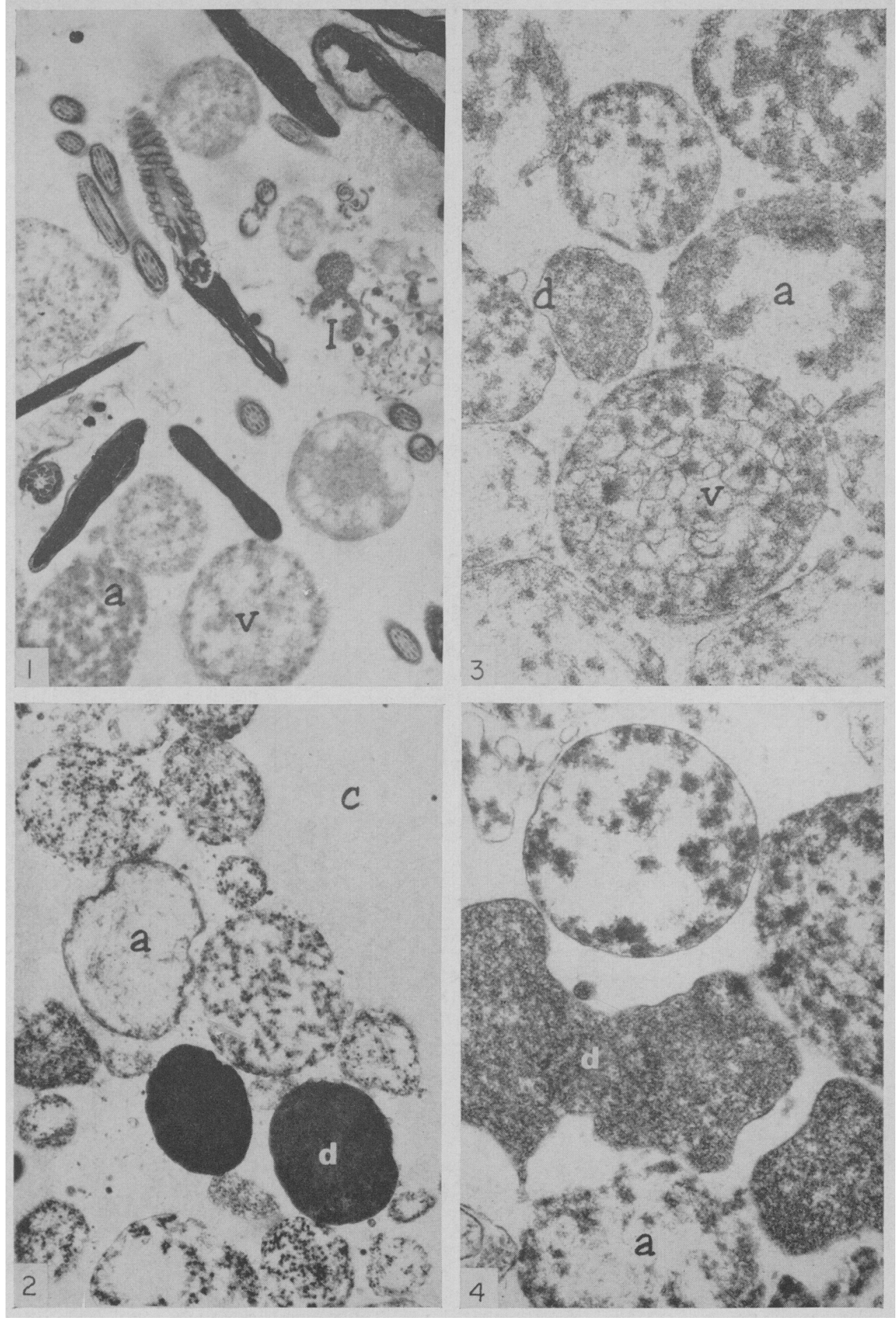

(Facing p. 196) 
PI. \TE 2

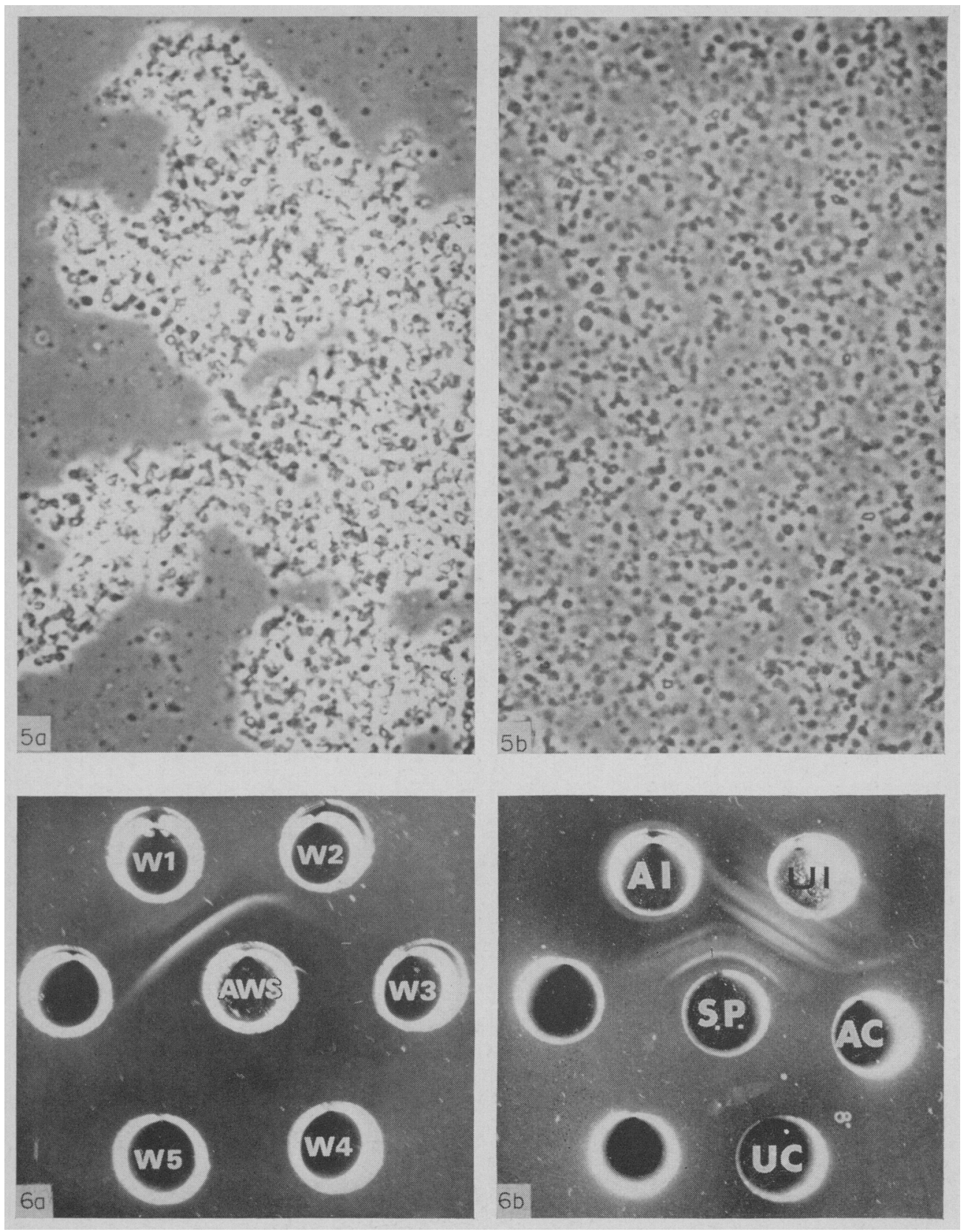

(Facing p. 197) 
In preliminary attempts to extract antigens, concentrated suspensions of washed particles were subjected to freeze-thawing, mechanical homogenization, sonic disintegration (Hypersonic Generator, General Radio Co., Cambridge, Massachusetts) and freeze-drying procedures. None of these attempts has consistently yielded supernatants that produce precipitin bands in immunodiffusion experiments.

To determine if seminal particles contain seminal plasma antigens, the particles were tested for capacity to absorb seminal plasma precipitating antibodies from anti-whole semen serum. The absorbing seminal particles were first washed five times in Hanks's solution and the washings tested by immunodiffusion to confirm removal of seminal plasma (Pl. 2, Fig. 6a). As seen in Pl. 2, Fig. 6b, antiserum to whole semen absorbed by washed particles produced, at most, only three to four precipitin bands when diffused against seminal plasma, whereas the unabsorbed antiserum at corresponding concentration produced eight to ten precipitin bands. A total of seven different serum samples from three different guinea-pigs was absorbed with particles and tested. In all experiments the absorbed sera produced no precipitin bands (dilute or unconcentrated sera) or fewer bands than the unabsorbed sera at corresponding concentration when diffused against seminal plasma.

From the above, it is evident that rabbit seminal particles possess the majority of soluble seminal plasma antigens. Since the rabbit spermatozoan surface antigenic complex includes seminal plasma antigens (Weil \& Rodenburg, 1962; see Metz, 1967; and Weil, 1967, for review), it seemed likely that spermatozoa and seminal particles might share some antigens. To examine for this, antisera to whole semen absorbed by washed particles were tested for sperm agglutinating action.

In six experiments absorbed sera which failed to agglutinate seminal particles did agglutinate spermatozoa. Accordingly, spermatozoa must possess surface antigens lacking in the seminal particles. However, the sperm agglutination by absorbed sera was weaker than by the unabsorbed sera at corresponding concentration. In one actual assay this was a four-fold difference in titre.

EXPLANATION OF PLATE 2

FIG. 5. Agglutination of rabbit seminal particles by antiserum. Semen from a vasectomized rabbit was centrifuged to remove seminal plasma. Seminal particles were then washed once in Hanks's solution and suspended in this solution. Two volumes of serum were mixed with one of particle suspension and photographed with phase optics after 30 min. (a) Seminal particle suspension plus anti-rabbit seminal solids guinea-pig serum. (b) Seminal particles plus control guinea-pig serum.

FIG. 6. Seminal plasma precipitating action of seminal particle absorbed serum. $0.96 \mathrm{ml}$ of sterile semen was centrifuged to sediment the seminal particles. These were washed five times in Hanks's solution ( $3 \mathrm{ml} /$ wash) to remove any traces of seminal plasma. The particles were then suspended in $0.38 \mathrm{ml}$ Hanks's solution. $0.07 \mathrm{ml}$ samples of particle suspension were mixed with $0.4 \mathrm{ml}$ serum (previously concentrated to one-half the original volume with Sephadex G-25) and centrifuged after $30 \mathrm{~min}$. This absorption was repeated two additional times. Unabsorbed serum samples were diluted correspondingly with Hanks's solution. (a) Effectiveness of particle washing procedure $W_{1} \rightarrow W_{5}$ : Supernatant Hanks's solution washes, one to five, removed from centrifuged particles. AWS, anti-whole rabbit semen guinea pig serum (unconcentrated). (b) Comparison of absorbed and unabsorbed serum. AI, Seminal particle absorbed, anti-whole semen guineapig serum; UI, unabsorbed anti-whole semen guinea-pig serum; AC, absorbed control guinea-pig serum; UC, unabsorbed control guinea-pig serum; SP, seminal plasma from whole semen. 
Absorbed and unabsorbed control sera served as negative standards. Evidently then, the seminal particles possess some, but not all, of the spermatozoan surface antigens.

The agglutinated spermatozoa were attached by the tails as well as heads so the spermatozoa-specific antigens (not present on seminal particles) are probably present on both heads and tails. Finally, when semen was treated with unabsorbed serum some particles appeared agglutinated to sperm heads but not to sperm tails. No appreciable particle-to-sperm agglutination was obtained with the particle-absorbed serum. This suggests that the antigens common to both sperm and particles are sperm head antigens.

The authors are indebted to Dr Joseph M. Branham, Department of Zoology, University of Hawaii for calling our attention to the nigrosine method; Dr David Smith, Department of Anatomy, University of Miami for use of the Philips EM 200; to Dr J. Garcia, (Director of the Division of Animal Care, Medical School, University of Miami) for his expert care of the animals and to Mrs Narelle McClung for her courtesy and superior technical assistance.

\section{REFERENCES}

BEDFord, J. M. (1965) Non-specific tail-tail agglutination of mammalian spermatozoa. Expl Cell Res. $38,654$.

Dotт, H. M. \& Walton, A. (1960) Effects of dilution and washing on ram spermatozoa studied by the flow dialysis technique. F. Reprod. Fert. 1, 350.

METz, C. B. (1967) Gamete surface components and their role in fertilization. In: Fertilization: Comparative Morphology, Biochemistry and Immunology, Vol. I. Eds. C. B. Metz and A. Monroy. Academic Press, New York.

WEIL, A. J. (1967) Antigens of the seminal plasma. F. Reprod. Fert. Suppl. 2, 25.

WeII, A. J. \& Rodenburg, J. M. (1962) The seminal vesicle as the source of the spermatozoa-coating antigen of seminal plasma. Proc. Soc. exp. Biol. Med. 109, 567. 